\title{
A Primer on Automating the Vertical Diffusion Cell (VDC)
}

\author{
Royal Hanson* and John Heaney \\ Hanson Research Corp., Chatsworth, CA, USA
}

e-mail:rhanson@hansonresearch.com

\section{INTRODUCTION TO VDC TESTING}

- - he vertical diffusion cell (VDC), aka "Franz Cell," is widely used for drug release-rate testing of semisolids and other topical formulations. Over the last several decades, the VDC has been used by researchers and formulators at most major pharmaceutical manufacturers worldwide. The VDC is recommended in the SUPAC-SS Guidelines (FDA 1997) for scale-up and postapproval changes for semisolids including creams, gels, and ointments.

The USP has introduced two new general chapters providing recommendations and guidelines for the testing of topicals and semisolids.

- <3> Topical and Transdermal Drug Products: Quality Tests (USP 35)

- $<1724>$ Semisolid Drug Products-Performance Tests (1S USP 36)

USP $<1724>$ in particular describes the VDC apparatus, including specifications and recommendations for setup and test procedures. Figures 1 and 2 illustrate the VDC (USP Model A) and its components.

As drug from the donor area permeates and diffuses through the membrane into the receptor solution, samples are withdrawn periodically and analyzed for concentration. The VDC may be sampled manually or automatically. A critical requirement in removing sample aliquots from the VDC is to concurrently introduce fresh receptor medium and avoid the creation of bubbles that may adhere to the membrane while doing so. This can be a tedious manual task for the analyst.

The first job of automation is to replicate human tasks with accuracy, reliability, and repeatability. The second job is to save the analyst time and hassle. It is appropriate therefore to review the manual steps in sampling from the VDC, and the challenges therein, before evaluating the advantages of automation.

\section{HOW VDC SAMPLING WORKS}

Figure 3 shows the components used in a manual VDC test system. These include a group of six VDCs mounted on a stir plate with speed control, a circulating waterbath to control temperature of the VDC, and a media replace beaker filled with deaerated replacement medium. For manual sampling, the analyst will typically use a sampling syringe to withdraw aliquots for analysis.

Some manual sampling routines (USP Models B and C) require first withdrawing a sample from the VDC and then manually replacing medium back into the receptor chamber. This can be troublesome for the analyst,

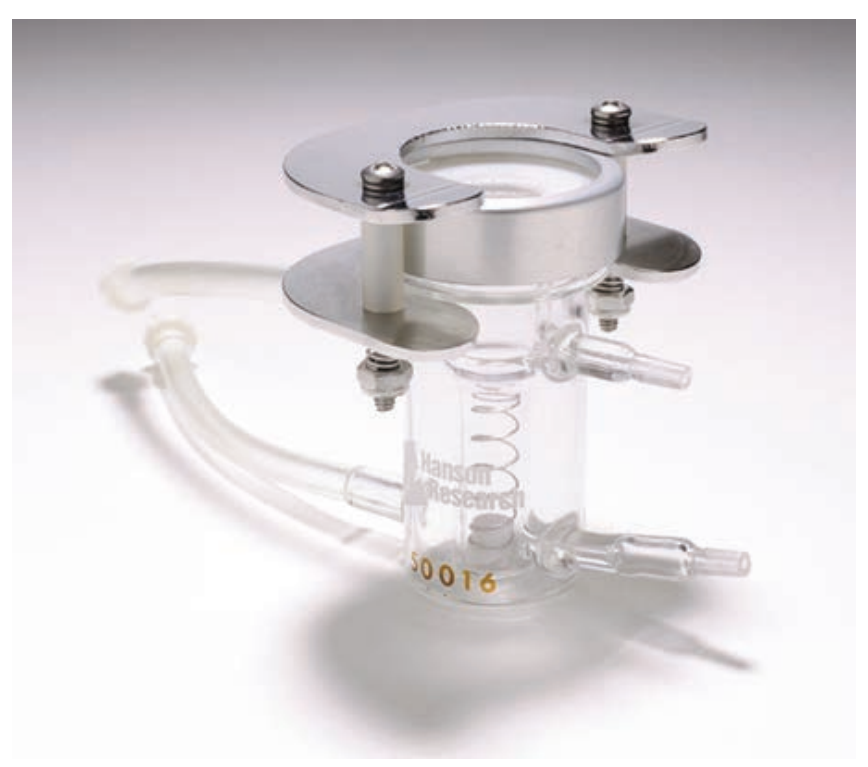

Figure 1. Vertical diffusion cell (VDC) assembly.

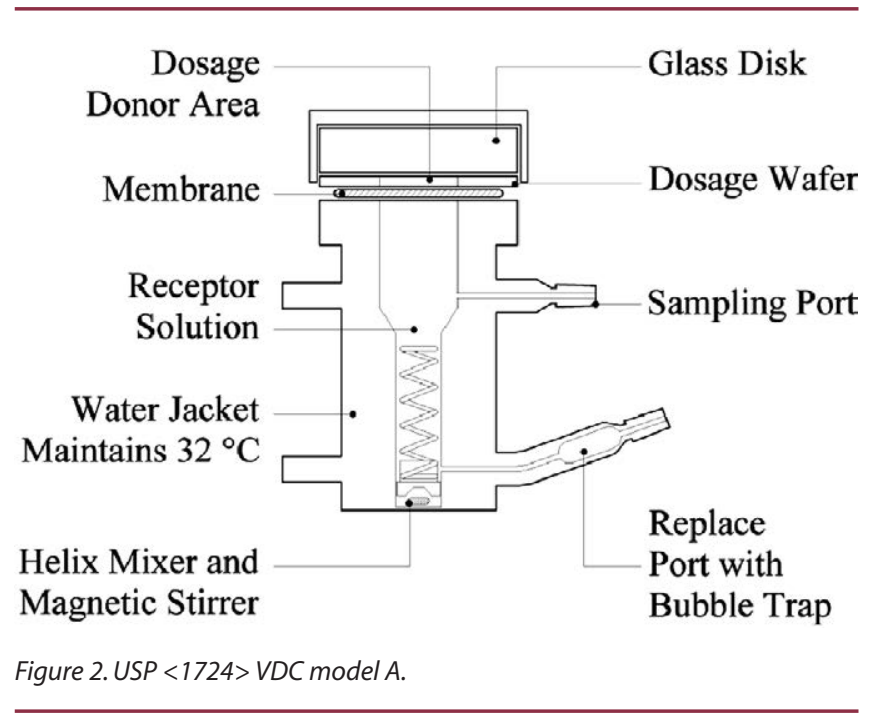

${ }^{*}$ Corresponding author. 


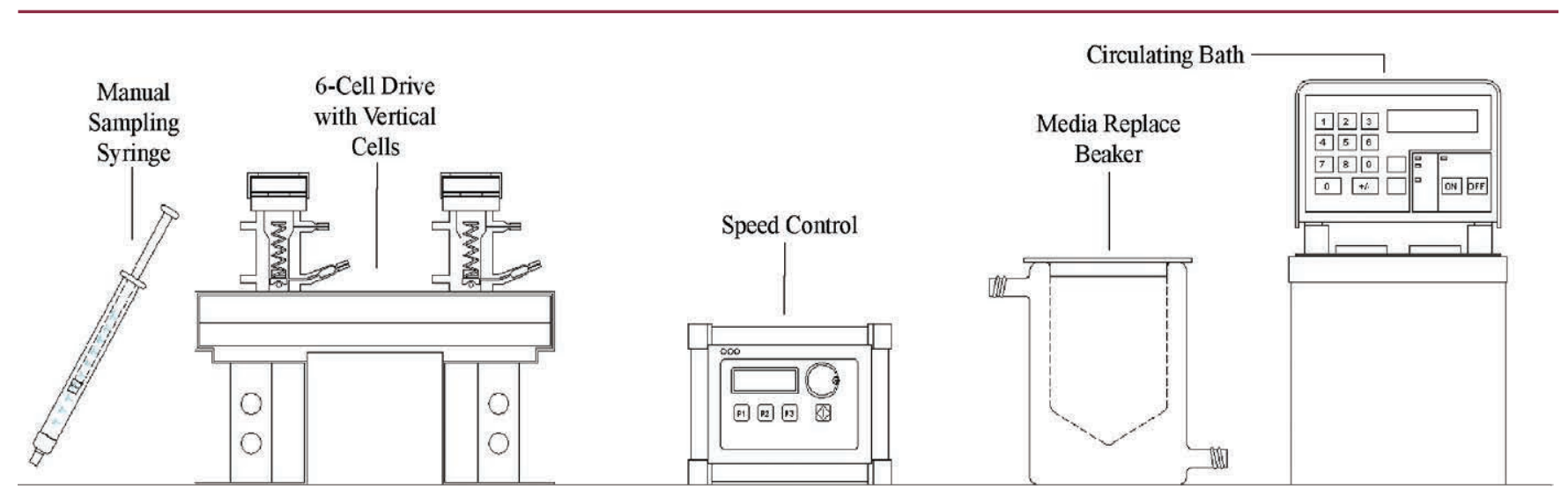

Figure 3. A manual VDC test system.
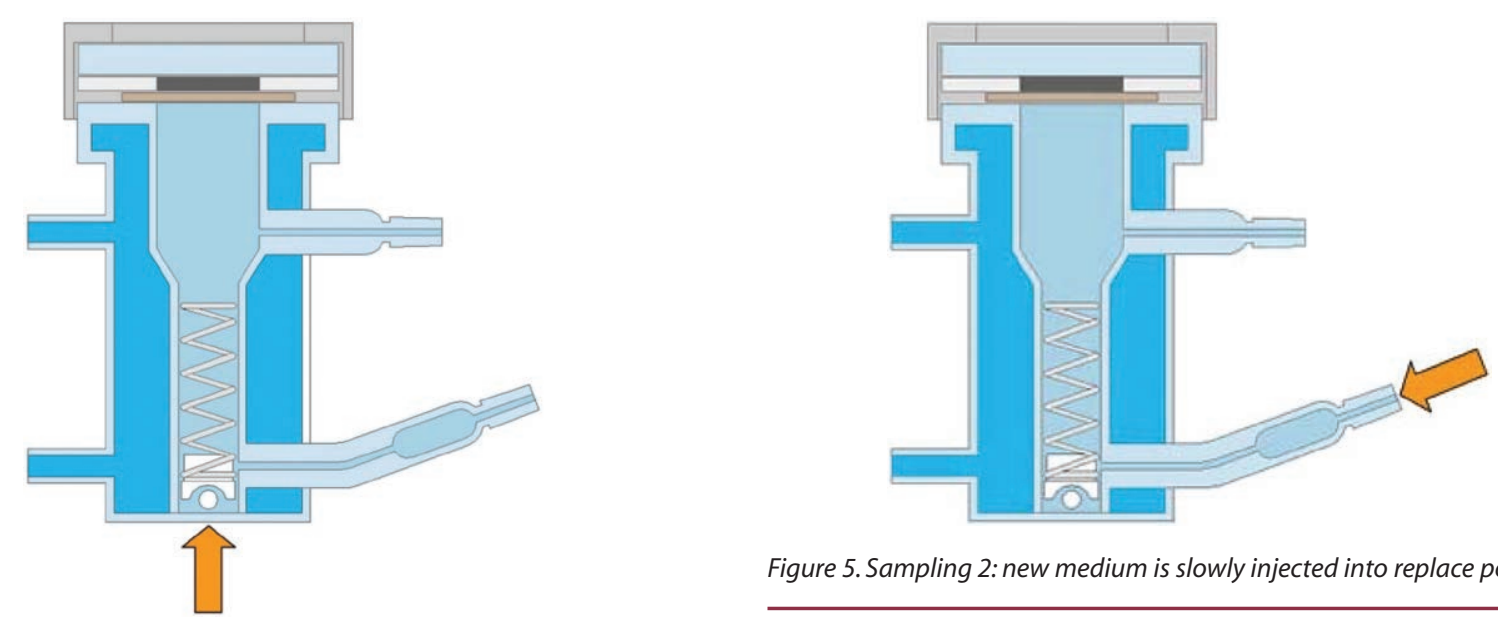

Figure 5. Sampling 2: new medium is slowly injected into replace port.

Figure 4. Sampling 1: stirrer stops $30 \mathrm{sec}$ prior to sample point.

particularly when trying to refill the cell without spilling or introducing bubbles into the medium. Bubbles that rise and adhere to the membrane will interfere with the membrane-liquid interface and will adversely skew test results.

A better method for sampling (whether manual or automated) is illustrated in Figures 4-7. With this procedure, the stirrer is first stopped to prevent media from mixing. Replacement medium is then slowly introduced into the bottom replace port of the VDC, while simultaneously a sample aliquot is forced out through the top sampling port of the VDC. The collected sample is then typically analyzed by HPLC for drug concentration.

There are several areas of concern when sampling from the VDC. First, the replacement medium volume (and resulting sample volume) must be accurate and held at a minimum to avoid any fresh medium diluting the concentrated receptor medium being removed as a sample. As a guideline, this may be a maximum $2.5 \mathrm{~mL}$ in a standard 7-mL VDC. 


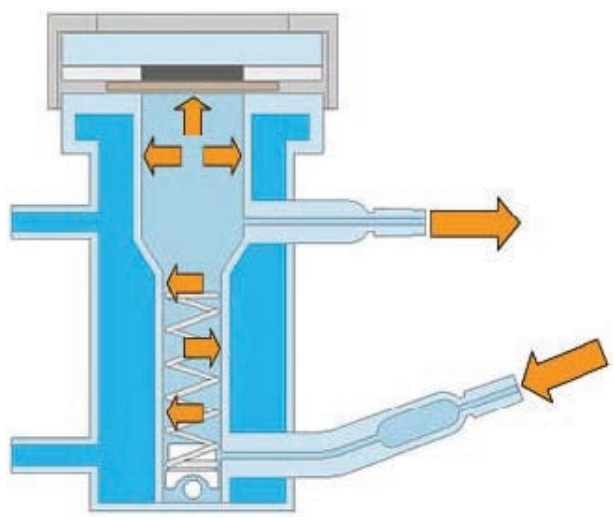

Figure 7. Sampling 4: sample aliquot is ejected from sample port.

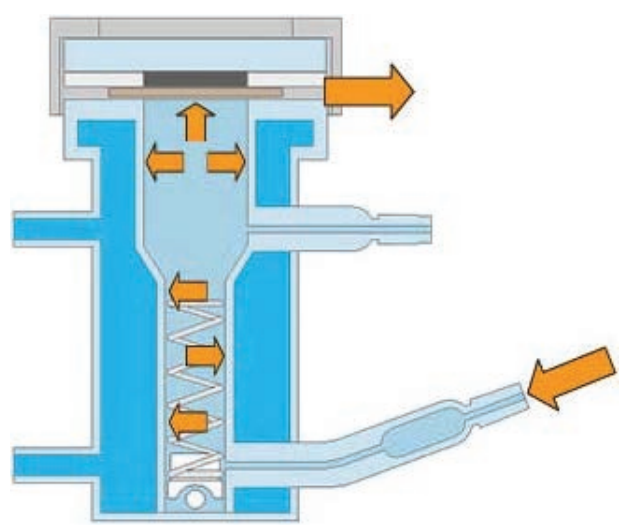

Figure 8. Problem area: avoid medium leakage from cell top.

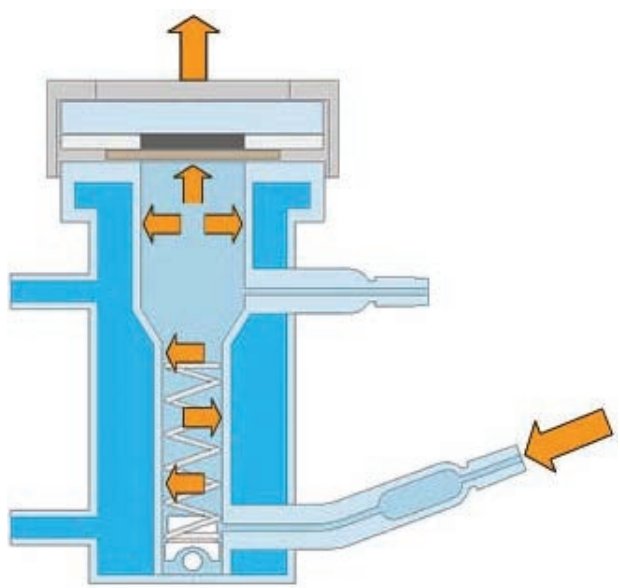

Figure 9. Problem area: avoid back diffusion up through membrane.

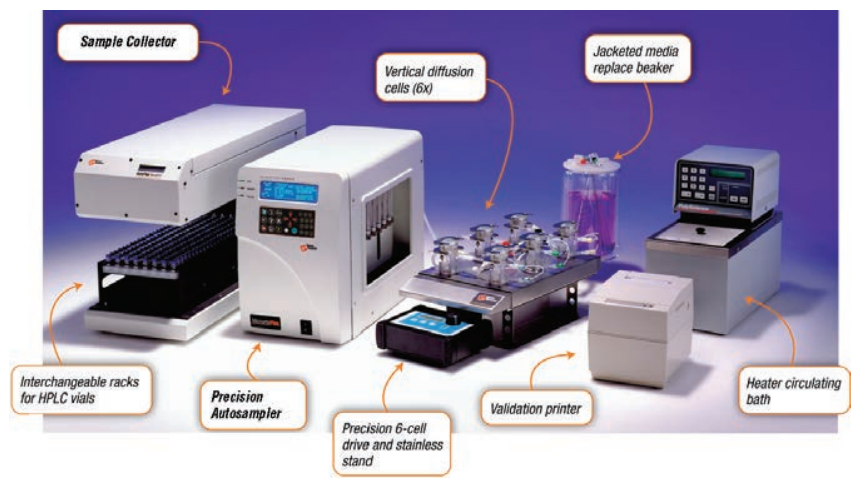

Figure 10. An automated VDC test system.

Figures 8 and 9 illustrate other areas of concern. When sampling, it should be noted that medium will follow the path of least resistance. The top donor compartment of the VDC must always be adequately clamped to the receptor compartment to avoid any medium leakage. It is also recommended to use an occluded (closed-top) VDC cell type to avoid any receptor medium from being forced back up through the membrane causing back diffusion.

Manually sampling from the VDC, collecting sample aliquots, and replacing receptor medium can be a timeconsuming task for the analyst. The SUPAC-SS recommended test is a multi-time point, 6 -h procedure, and other test methods require sampling overnight or even over the course of several days. An automated procedure can therefore offer great value and time savings.

\section{HOW AN AUTOMATED VDC SYSTEM WORKS}

Several instrument systems for automating VDC testing have been employed throughout the industry (Hanson, Logan, PermeGear, and others). Peristaltic pumping systems, although relatively low cost, have inherent problems with fluctuating flow rates, drug absorbance or leaching from plastic tubing, and related maintenance issues. A rugged and robust automated sampling system can overcome these instrument deficiencies.

Figure 10 shows a precision syringe pump sampling system designed for automating VDC testing. This autosampling and collection system includes chemically inert valves and flow tubing and readily adapts to the manual VDC test system described above (Figure 3). This system provides unattended test runs and includes automatic collection of samples in sealed-septum HPLC or UPLC vials for later analysis.

Figure 11 illustrates the flow path of how an automated VDC system works. The diagram shows one VDC, although groups of six VDCs are typically tested in parallel. Up to three groups of VDCs may be tested in sequence with this system.

The following steps outline how an automated VDC system works.

1) The system is set up by filling each VDC with heated 


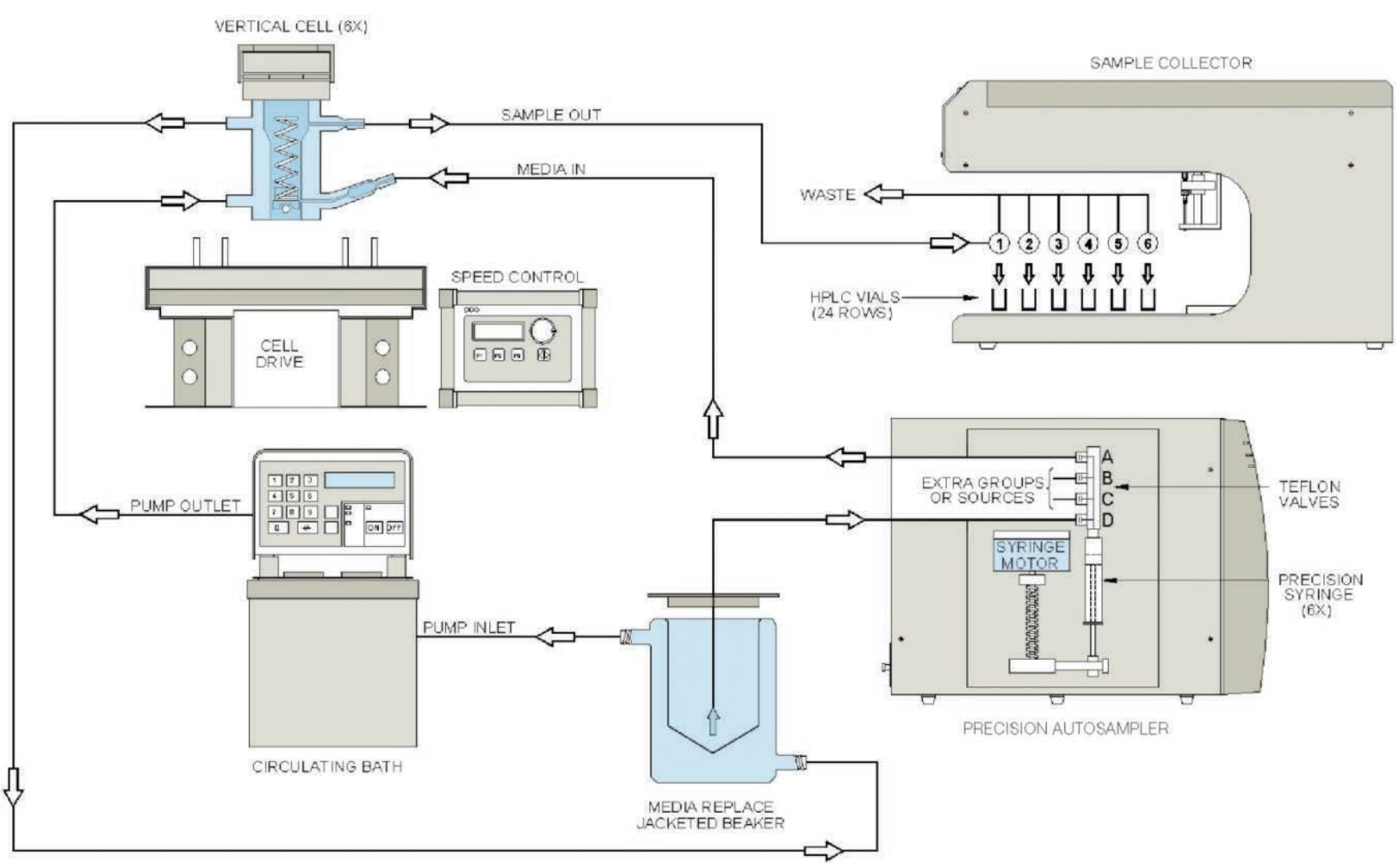

Figure 11. Automated VDC system flow diagram.

deaerated receptor solution from the media beaker. A measured volume of drug formulation is then placed on the membrane in the cell donor chamber to start the test.

2) The autosampler saves protocols for sampling volumes and time points including advanced routines for cell dilution and other requirements. A given protocol is activated to initiate the start, and the analyst returns to other priorities.

3) On program command, the stirrer stops $30 \mathrm{sec}$ prior to the sample point, and a selected aliquot of fresh medium is injected from the precision syringe pump into the capillary media replace port of the VDC. This forces an exact, equal amount from the cell to be extracted through the sample port to the collector.

4) Sample aliquots are collected and archived in sealedseptum HPLC vials in the collector. When each sampling point is complete, the stirrer starts again to restore homogeneity.

5) A rinse cycle just before each sampling cycle ejects contents of the sample tube to waste. This cleans the sample lines prior to sampling and collection.

6) This sampling and collection sequence continues for each programmed time point until the end of the test. A validation printer provides an ongoing status report of all test operating conditions.

7) The use of advanced "scripting" allows for special protocol routines, including cell dilution at selected time points.

8) Throughout the test, the heater circulating bath maintains controlled temperature in the media replacement beaker and each VDC (typically $32^{\circ} \mathrm{C}$ for skin permeation studies).

9) Collected samples archived in interchangeable racks may be removed at the convenience of the analyst for HPLC and other method analyses.

10) The automated sampling and collection routines outlined above run virtually unattended throughout the entire diffusion test. For long tests (e.g., 6-10 h or more), it is recommended to replenish the media replacement beaker with freshly deaerated medium.

The pharmaceutical industry has recognized the value in cost savings and efficiency by automating countless testing procedures. Automated dissolution systems are popular throughout R\&D and QC laboratories worldwide. Automating the VDC test for analyzing the release-rate characteristics of semisolids and other topical preparations provides similar benefit. 